\title{
AIE-nanoparticle assisted ultra-deep microscopy in the in vivo mouse brain under 1300-nm excitation
}

\author{
Dong-yu Li, ${ }^{\#, a, b, c, d}$ He-qun Zhang, ${ }^{\#, b, e}$ Lina L. Streich, ${ }^{c, f}$ Ping Lu, ${ }^{\mathrm{g}}$ Ling Wang, ${ }^{\mathrm{c}}$ Robert Prevedel*c and Jun Qian*b
}

a. Britton Chance Center for Biomedical Photonics, Wuhan National Laboratory for Optoelectronics, Huazhong University of Science and Technology, Wuhan, Hubei, China.

b. State Key Laboratory of Modern Optical Instrumentations, Centre for Optical and Electromagnetic Research, College of Optical Science and Engineering, International Research Center for Advanced Photonics, Zhejiang University, Hangzhou, China.

c. Cell Biology and Biophysics Unit, European Molecular Biology Laboratory (EMBL), Heidelberg 69126, Germany.

d. MoE Key Laboratory for Biomedical Photonics, Huazhong University of Science and Technology, Wuhan, Hubei, China.

e. Zhejiang University Interdisciplinary Institute of Neuroscience and Technology, The Second Affiliated Hospital, School of Medicine, Zhejiang University, Hangzhou, Zhejiang

f. Candidate for Joint PhD degree from EMBL and Heidelberg University, Faculty of Biosciences, Heidelberg 69126, Germany.

g. State Key Lab of Supramolecular Structure and Materials, Jilin University, Changchun 130012, China.

\#. These authors contributed equally to this work.

*Corresponding authors: Jun Qian, Email: qianjun@zju.edu.cn

Robert Prevedel, Email: prevedel@embl.de

\begin{abstract}
Aggregation-induced emission nanoparticles serve as promising fluorescence probes for multi-photon excitation microscopy due to the large absorption cross-section at NIR-IIb region. Here we present organic AIE nanoparticles that feature high aborption cross-section under three-photon excitation. We show that these enable ultra-deep NIR-Ila excited three-photon imaging in the in-vivo mouse brain.
\end{abstract}

\section{Introduction}

Modern optical imaging techniques have attracted wide attention in the field of biomedical research due to their key advantages of high resolution and low invasiveness, and thus provide a powerful tool for in vivo monitoring of biological structure and function. ${ }^{1-3}$ For example, fluorescence imaging can be utilized to visualize neural activity, vasodilation and -constriction, and blood oxygenation in brain. ${ }^{4-6}$ However, while many optical techniques, including basic fluorescence imaging, permit imaging at superficial layers of tissues, imaging deeper into mammalian tissues is challenging. ${ }^{4,7,8}$ While confocal microscopy obtains high 3D resolution, the attenuation of tissue to visible (fluorescence) light caused by absorption and scattering limits the depths of traditional confocal imaging to only a few dozens of microns. Compared to confocal microscopy, two-photon fluorescence microscopy (2PM) significantly extends the imaging depth by employing near infrared-I (NIR-I, 700-900 nm) excitation light. Here, the non-linear excitation confines the fluorescence excitation and thus permits detection of scattered as well as ballistic signal photons. Multi-photon excitation thus enables imaging from a few hundred microns to a millimeter below the surface of scattering, mammalian tissues. ${ }^{9-13}$ To further increase the achievable imaging depth, three-photon fluorescence microscopy (3PM) has been explored and developed in the past. ${ }^{14-16}$ 3PM uses NIR-II (900-1700 nm) excitation light, ${ }^{17,18}$ which leads to reduced scattering and overall attenuation and thus to increased penetration depth in tissue. Furthermore, the fluorescence of three-photon excitation falls off as $1 / z^{4}$ (where $z$ is the distance from the focal plane), which yields less out-of-focus background and therefore better signal-to-background ratio (SBR) at significant tissue depths. ${ }^{19}$ Despite these clear advantages, 3PM places stringent requirements on the fluorescence probes in terms of high three-photon absorption cross section, quantum yield, photostability as well as biocompatibility. These render most traditional fluorescence probes such as proteins or small molecule dyes challenging for practical 3PM. Therefore, there exists an urgent need to develop novel probes that meet those requirements and are optimized for 3PM and its NIR-II excitation. ${ }^{20,21}$

Due to their easy modification and remarkable biocompatibility, numerous organic molecules with high quantum yields have been used as fluorescent probes in biological imaging in recent years. ${ }^{22}$ However, most of the organic dyes suffer from aggregation-caused quenching (ACQ) effect due to their planar shaped molecular structure, leading to reduced fluorescence when in the (nano)aggregated state or at a relatively high molecular concentration. ${ }^{23,24}$ The ACQ effect not only limits the brightness of such molecules for biological imaging, but also 
restrict their photostability, because organic fluorophores in isolated state at molecular level are more easily photobleached under laser excitation, ${ }^{25,26}$ especially at the ultra-high pulse-energy femtosecond ( $\mathrm{fs}$ ) laser excitation which is typically used in 3PM. ${ }^{14-19}$

In contrary to the ACQ effect, aggregation-induced emission (AIE) phenomenon can lead to high amounts of fluorescence in the aggregate or solid state. ${ }^{27-29}$ This phenomenon exists in propeller shaped organic molecules which are non-emissive or provide weak fluorescence in benign solvents, but provide orders-of-magnitude higher fluorescence in aggregated form. To exploit this effect, thousands of AIE molecules can be doped into one nanoparticle (NP) to improve their overall absorption cross section, quantum yield and photobleaching resistance, ${ }^{30}$ suggesting AIE-NPs to be excellent three-photon fluorescent probes. In addition, due to the structural flexibility, AIE fluorogens (AIEgens) can in principle be designed for large multiphoton absorption cross sections. For instance, the three-photon absorption cross section of TBDTT is $1.92 \times 10^{-81} \mathrm{~cm}^{6} \mathrm{~s}^{2}$ at $1600 \mathrm{~nm}{ }^{31}$, DCDPP-2TPA is $2.95 \times 10^{-79} \mathrm{~cm}^{6} \mathrm{~s}^{2}$ at $1550 \mathrm{~nm}, 32$ and TPATCN can reach $5.77 \times 10^{-79} \mathrm{~cm}^{6} \mathrm{~s}^{2}$ at $1550 \mathrm{~nm},{ }^{33}$ which is comparable to those of bright quantum dots (QDs) ${ }^{14}, 34$, and far higher than those of common fluorophores, such as fluorescein and GFP $\left(\sim 10^{-83} \mathrm{~cm}^{6} \mathrm{~s}^{2}\right) .{ }^{35}$ Accordingly, AlEgens have shown promising performance in high-resolution deep-tissue imaging when excited by three-photon. 31-33

It is generally thought that the region of NIR-IIb (1500-1700 nm) is more suitable than NIR-Ila (1300-1400 nm) for deep-tissue imaging, because of lower scattering. ${ }^{36-38}$ However, overall tissue absorption is rather strong in the NIR-IIb window because of the water's absorption spectrum (Fig. 1), ${ }^{15}, 39$ which can cause severe heating-related photodamage and thus limit the maximum excitation laser power for physiological imaging. Altogether, we reason that NIR-Ila excitation might be optimal for in-vivo deeptissue imaging applications ${ }^{40-42}$ despite the slightly enhanced scattering in this wavelength window.

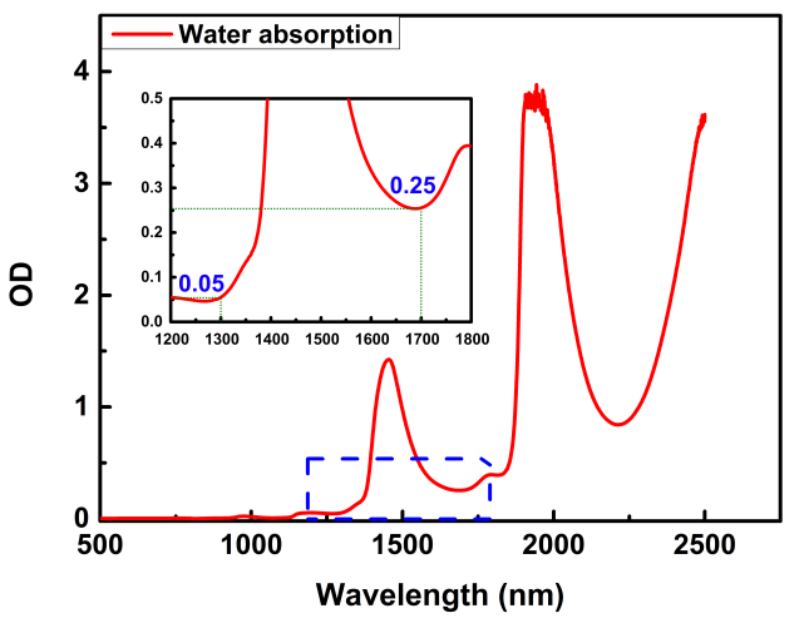

Fig. 1 Absorption spectrum and attenuation of water from $500 \mathrm{~nm}$ to $2500 \mathrm{~nm}$. The water depth was set to $2 \mathrm{~mm}$, corresponding to the working distance of typical physiology objectives. Inset shows expanded view of blue dashed box area.

In this work, we investigated the performance of AIE NPs optimized for NIR-Ila excitation as three-photon fluorescent probes in deep-tissue imaging. In particular, we chose TPATCN molecules which have previously been shown to exhibit a high three-photon absorption cross section at $1550 \mathrm{~nm}^{33}$. After doping them into organic NPs we characterized them for NIR-Ila 3P-excitation at $1300 \mathrm{~nm}$. Our results show that the three-photon absorption cross section of TPATCN at $1300 \mathrm{~nm}$ is actually increased compared to $1550 \mathrm{~nm}$. Based on this we demonstrated the capabilities of our TPATCN-based AIE NPs for deep-tissue brain imaging. Utilizing a custom-built three-photon microscope, we obtained high signal-to-background ratio (SBR) and an overall imaging depth of $\sim 1.4$ $\mathrm{mm}$ when imaging sub-cortical vasculature in the in-vivo mouse.

\section{Results and discussion}

Preparation and optical characterization of TPATCN NPs 
As illustrated in Fig. 2, the TPATCN@F-127 NPs were synthesized via a modified nanoprecipitation method which resulted in a hydrophobic TPATCN molecules core and a amphiphilic Pluronic F-127 molecule matrix. The NPs could be dispersed clearly and stably in water, and the overall method was optimized for simplicity and reliability.

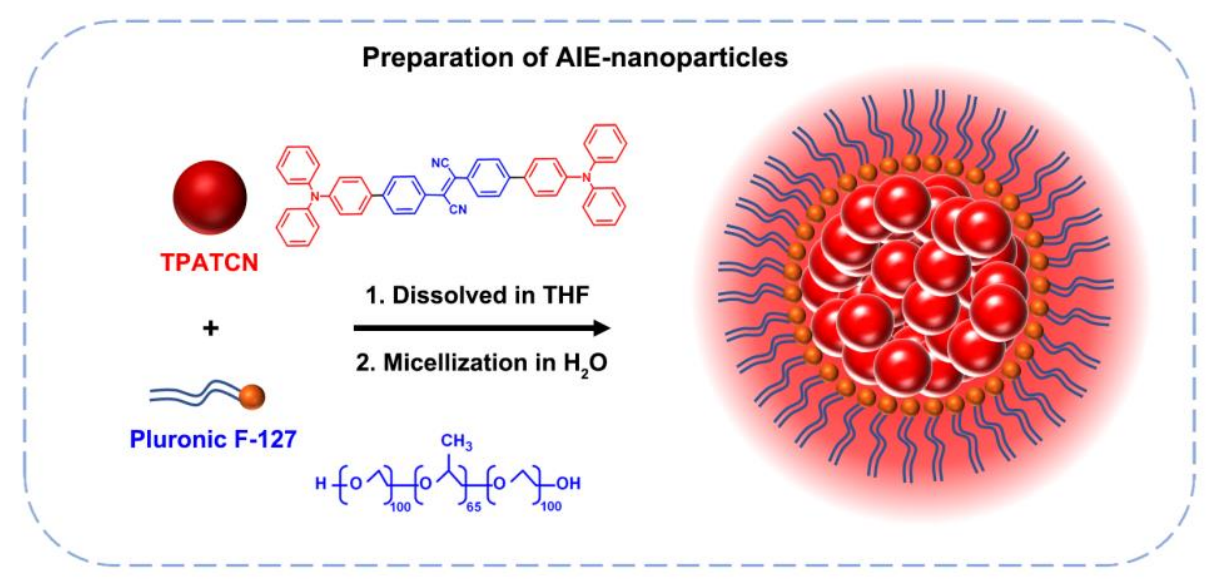

Fig.2 Chemical structures of TPATCN and Pluronic F-127, and schematic illustration of the synthesis of TPATCN@F-127 nanoparticles.

Fig. 3a shows the absorption (blue line) and one-photon fluorescence spectra (red line) of TPATCN@F-127 NPs in aqueous dispersion. We found the absorption peak of TPATCN@F-127 NPs at 444 nm with deep-red fluorescence generated at a peak wavelength of $648 \mathrm{~nm}$.

The nonlinear optical characters of TPATCN@F-127 NPs were measured under 1300-nm excitation. As shown in Fig. 3b, both TPATCN in $\mathrm{CHCl}_{3}$ solution (purple line) and in NPs dispersed in water (red line) could generate fluorescence under the excitation of $1300-\mathrm{nm}$ (fs) laser. The emission peak of the NPs was observed at $640 \mathrm{~nm}$, which is effectively unaltered compared to one-photon fluorescence. Compared to the NPs, TPATCN in $\mathrm{CHCl}_{3}$ solution generated NIR-I fluorescence under the same fs-laser excitation, with a peak wavelength of $744 \mathrm{~nm}$. We reason that this red shift is due to the smaller polarity of $\mathrm{CHCl}_{3}$.

The excitation power dependence relationship of the fluorescence from TPATCN@F-127 NPs was measured to identify the nonlinear emission under the excitation of $1300 \mathrm{~nm}$. The NPs in aqueous dispersion was filled in a capillary glass tube and further imaged by a home-built microscopy system. As shown in Fig. 3c, the fluorescence intensity was rapidly enhanced with the increase of laser power. The logarithm of the fluorescence intensity of TPATCN NPs showed a good linear relation to the logarithm of the excitation power, with a slope of 2.83 , which is close to 3 . Taking the emission spectrum into account, this suggests that threephoton fluorescence should be the main nonlinear optical process under $1300-\mathrm{nm}$ fs excitation.

Next, we studied the three-photon absorption cross section of TPATCN. Its three-photon absorption cross section has been measured in previous work to be $5.77 \times 10^{-79} \mathrm{~cm}^{6} \mathrm{~s}^{2}$ at $1550 \mathrm{~nm} .^{2}$ This allowed us to calculate the three-photon absorption cross section at $1300 \mathrm{~nm}$ via comparison of the fluorescence intensity under excitation of these two wavelengths with the same power, pulse length and repetition rate. As shown in Fig. 3d, the three-photon fluorescence was much stronger at 1300-nm excitation than that of $1550-\mathrm{nm}$ excitation, and the three-photon absorption cross section at $1300 \mathrm{~nm}$ was thus inferred as $1.93 \times 10^{-78} \mathrm{~cm}^{6}$ $\mathrm{s}^{2}$, which is the highest value among reported organic dyes, making it a promising optical probe for NIR-Ila excited 3PM.

In addition to the good non-linear optical characters, the TPATCN@F-127 NPs have been reported to have high chemical stability (the absorption and emission spectra remained unchanged in various $\mathrm{pH}$ values), high photostability (the fluorescence remained stable under the 1550-nm fs laser irradiation) and high biocompatibility (the injection of the NPs won't cause inflammation or abnormalities on their major organs, which had experienced the accumulation and excretion of the NPs). ${ }^{28}$ Taken together, TPATCN@F-127 NP are an ideal probe for in vivo deep-tissue 3PM under NIR-Ila excitation, which has potentially low photo-toxicity to the animal. 

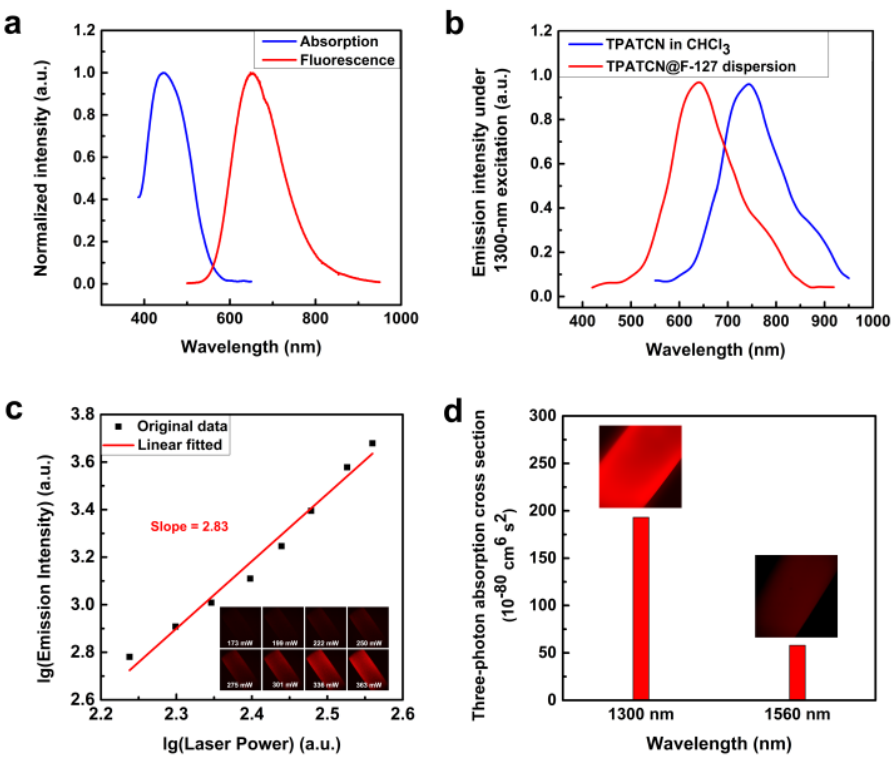

Fig.3 (a) absorption and one-photon fluorescence spectra of TPATCN@F-127 NPs in aqueous dispersion. (b) Emission spectra of TPATCN in $\mathrm{CHCl}_{3}$ solution and TPATCN NPs in aqueous dispersion under $1300-\mathrm{nm}$ fs excitation. (c) Relationship of excitation power and fluorescence from TPATCN@F-127 NPs under 1300-nm excitation. The insets are the 3PM images of a glass capillary filled with TPATCN NPs in aqueous dispersion with various excitation power. The power was measured before passing through the microscope optics. (d) Three-photon absorption cross sections at $1300 \mathrm{~nm}$ and $1550 \mathrm{~nm}$. The insets are the 3PM images a glass capillary filled with TPATCN NPs in aqueous dispersion under the excitation of $1300 \mathrm{~nm}$ and $1560 \mathrm{~nm}$ with the same pulse length $(200 \mathrm{fs})$, repetition rate $(400 \mathrm{kHz})$ and average power (20 $\mathrm{mW}$ after objective), respectively.

\section{NIR-I excited in vivo deep-tissue 3PM using TPATCN NPs}

In order to perform 3PM, we utilized a self-built dual channel laser scanning microscope equipped with a tunable fs laser as shown in Fig. 4. The light source consisted of a 1040-nm fs pump laser and a noncollinear optical parametric amplifier which was tuned to $1300 \mathrm{~nm}$ excitation wavelength for this study. To maintain short, $50 \mathrm{fs}$ pulses at the sample plane, we built a single-prism pulse compressor. ${ }^{43}$ This ensured a high peak pulse energy which is critical for high 3P excitation efficiency. In this work, only the red channel was used.

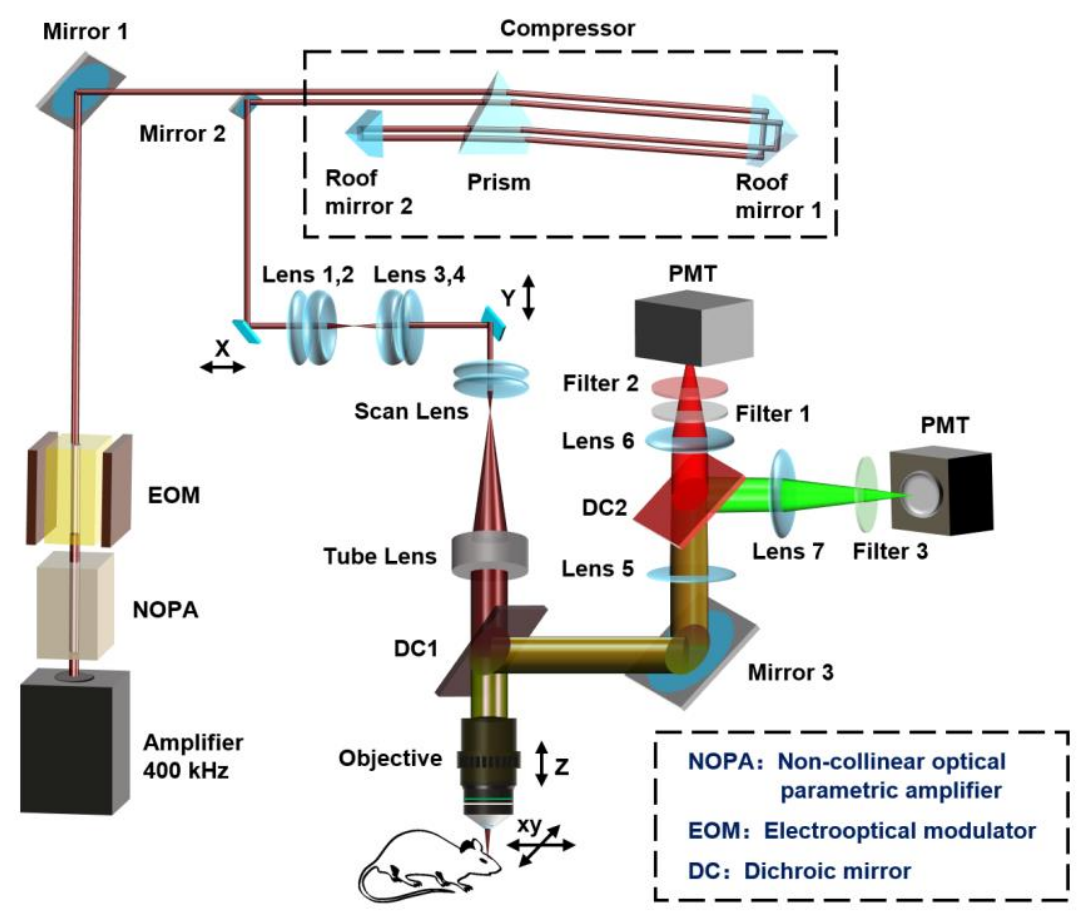

Fig. 4 Schematic illustration of the three-photon microscopy system. 
bioRxiv preprint doi: https://doi.org/10.1101/2020.12.25.424420; this version posted December 26, 2020. The copyright holder for this preprint (which was not certified by peer review) is the author/funder. All rights reserved. No reuse allowed without permission.

Fig. 5 shows the results of TPATCN@F-127 assisted in vivo brain 3PM. Due to the promising absorption cross section and biocompatibility, the signals of the NPs were bright and uniform in the vasculature. The unique properties of the NPs enabled highcontrast and deep tissue imaging of the brain vasculature from the surface into deep cortical and sub-cortical regions. The 3PM allowed us to resolve individual microvasculature with diameter as small as a few micrometers even in sub-cortical brain tissue, i.e. below the highly scattering corpus collosum layer. The high SBR enabled 3D vessel reconstruction to depth up to $1360 \mu \mathrm{m}$, which represents the deepest in NIR-Ila excited in vivo 3PM at this excitation wavelength to date.
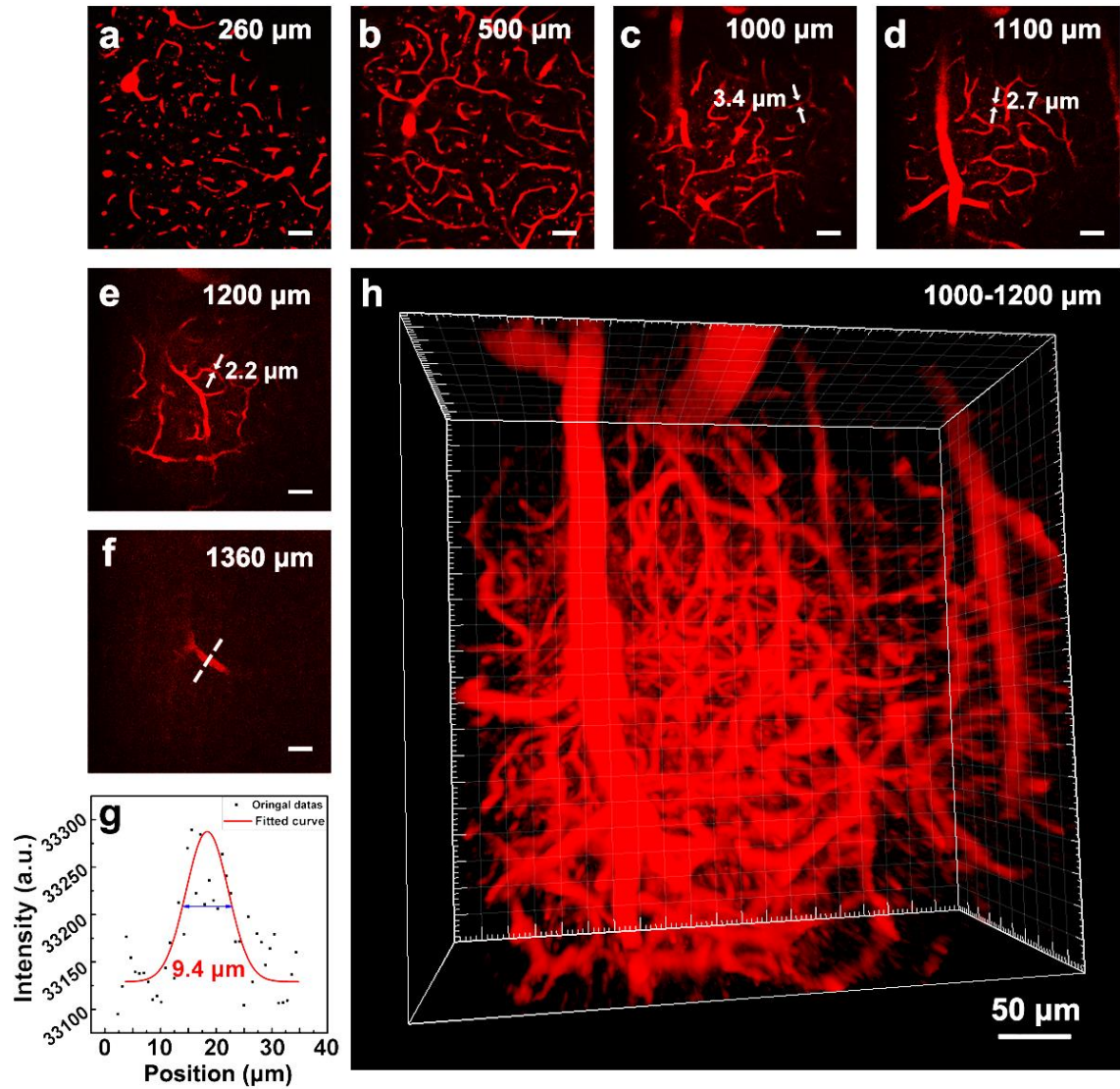

Fig. 5 (a)-(f) 3PM images at various brain depth. The values beside the white arrows in (c)-(e) represent the measured diameters of blood vessels ( $g$ ) Line plot of dashed line in (f). (h) 3D reconstruction of cortical vasculature in the depth range from $1000-1200$ $\mu \mathrm{m}$.

The SBRs at different depths are shown in Fig. 6. The exceptionally high three-photon absorption cross section and QY of TPATCN@F-127 NPs yielded an excellent SBR of up to 24 with only $3.5 \mathrm{~mW}$ of laser power after the objective. With the same excitation power, the SBR remained high $(>10)$ until $\sim 500 \mu \mathrm{m}$ after which the excitation laser power was continuously increased (to a maximum of $35 \mathrm{~mW}$ ) to maintain the signal intensity. Doing so maintained SBRs of 10 up to $1000 \mu \mathrm{m}$, thanks to the low background associated with 3P excitation. Below $1000 \mu \mathrm{m}$, the SBR started to drop due to the limited remaining excitation power at this tissue depths. Nevertheless, the SBR at $1360 \mu \mathrm{m}$ was still measured to be 2.6. Even deeper imaging (down to SBR 1) would thus have been possible theoretically, but practical limitations associated with the craniotomy prevented us from lowering the objective further.

Deep-tissue 3PM relies on high pulse energies to excite three-photon fluorescence. Specifically, the peak pulse power after the objective was as high as $1.75 \mathrm{MW}$ (average power $=35 \mathrm{~mW}$, pulse width $=50 \mathrm{fs}$, pulse frequency $=400 \mathrm{kHz}$ ) when imaging below $1000 \mu \mathrm{m}$. Here the comparatively low tissue, i.e. water, absorption of $1300 \mathrm{~nm}$ compared to other NIR wavelengths (e.g. 1700nm - see Fig. 1) was crucial in order to prevent photodamage and heating effects which could alter the physiology of the sample. In our experiments, we continuously monitored the breathing and heartbeat of the mouse and found no abnormalities during the imaging process. 

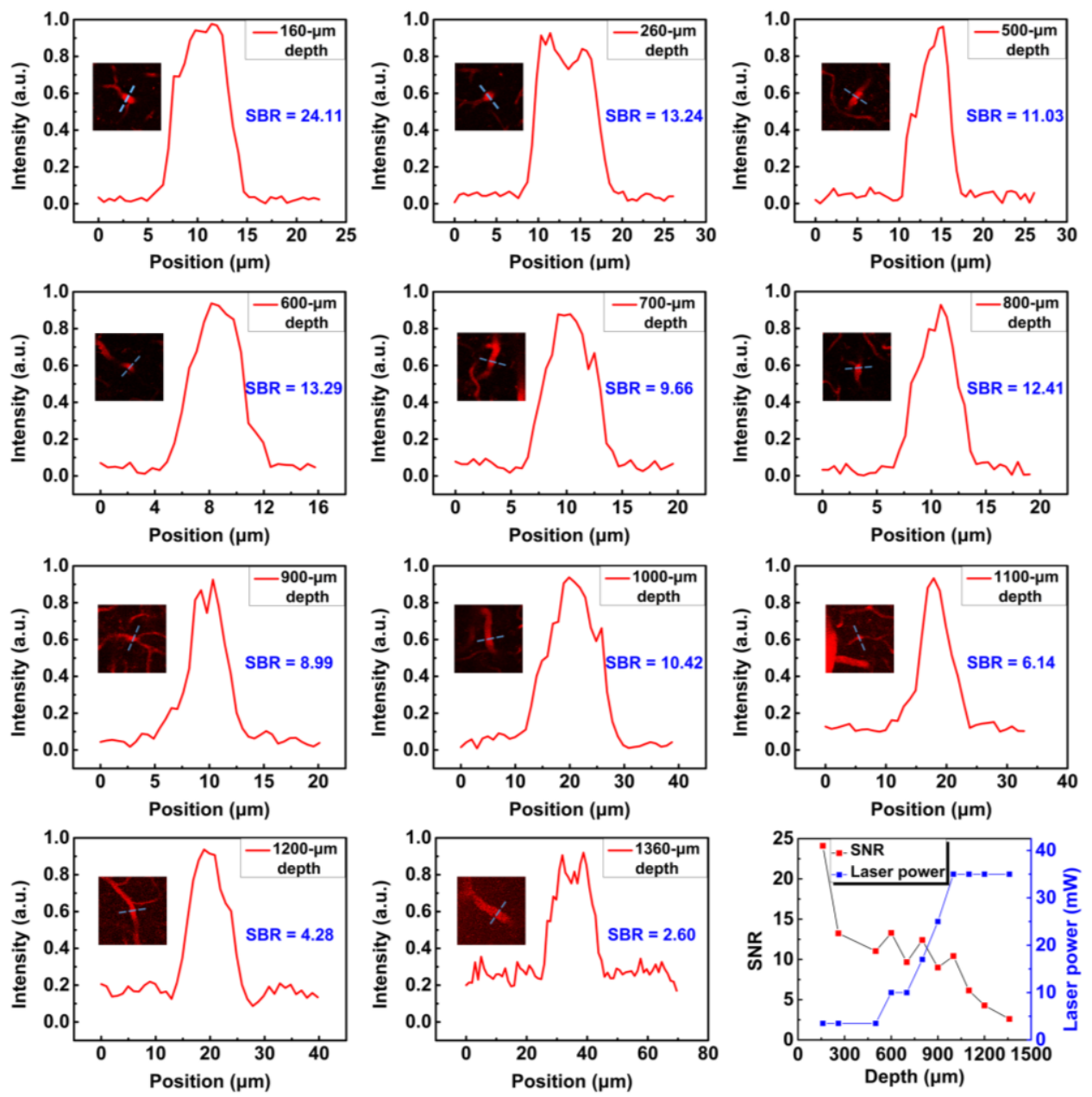

Fig. 6 Representative SBR of in vivo 3PM at each imaging depth of brain. Insets show the ROIs used for SBR calculation (see dashed lines).

\section{Conclusions}

AIEgens are emerging and promising fluorescence probes for 3PM for in vivo deep-tissue imaging, due to their high three-photon absorption cross section, high QY in NPs, high photobleaching resistance and high biocompatibility. However, previous work employed excitation lasers exclusively in the NIR-Ilb region, where tissue scattering is minimal but water absorption cannot be ignored. Since 3PM requires high, MW-scale peak pulse powers, such NIR-Ilb excitation might introduce nonnegligible heatingrelated photodamage in live animal. Compared to NIR-IIb, NIR-Ila is much more favourable in this respect and can thus be argued to be more biocompatible. As AIE probes optimized for NIR-Ila excited 3PM have not been reported, in this work, we utilized a typical AIE molecule, TPATCN doped into NPs, and explored its capabilities for three-photon microscopy probes. We found that the TPATCN@F-127 NPs could emit bright three-photon fluorescence under the excitation of 1300-nm (NIR-Ila) fs laser. Remarkably, the three-photon absorption cross section at $1300 \mathrm{~nm}$ was measured to be as high as $1.93 \times 10^{-78} \mathrm{~cm}^{6} \mathrm{~s}^{2}$, which is the highest among the organic materials reported in the literature. We demonstrated the capabilities of our TPATCN NPs by performing in vivo deep-tissue 3PM experiment. Here the NPs exhibited excellent performance and the brain's microvasculature could be clearly resolved up to a depth of $\sim 1.4 \mathrm{~mm}$, amongst the best reported in the literature. In particular, the SBR remained remarkably high in sub-cortical regions of more than $1 \mathrm{~mm}$ depth. Because of the low tissue absorption at 1300nm, no changes in blood vessel morphology, respiration or heartbeat were observed during the imaging process despite the high pulse power of the excitation laser. Thus, TPATCN NPs assisted NIR-Ila excited 3PM holds great potential for sub-cortical brain investigation with low heatingrelated photodamage.

\section{Experimental section}

Materials and instruments 
The TPATCN were synthesized via the reported protocol. Other chemicals were purchased from Sigma-Aldrich Co., Ltd. The absorption spectra were recorded from 400 to $2500 \mathrm{~nm}$ with a spectrophotometer (Cary 5000, Agilent Technologies Inc., USA), at room temperature. The one-photon fluorescence spectrum was recorded using a fluorescence spectrophotometer (F-2500, HITACHI, Japan) with a xenon lamp (excitation range, 300-800 $\mathrm{nm}$ ).

\section{Synthesis of TPATCN@F-127 NPs}

TPATCN@F127 NPs were synthesized through a modified nanoprecipitation method. ${ }^{25,44}$ Briefly, 1 mg TPATCN molecule and 10 mg F-127 were solved in $1 \mathrm{~mL}$ of THF. The THF solution was then added into $1 \mathrm{~mL} \mathrm{DI}$ water drop by drop with stirring. Next, the solution was evaporated using a rotary evaporator for $2 \mathrm{~min}$ at room temperature. Since THF has a lower boiling point than water, this step could remove the THF, while the water was remained. In this way, TPATCN@F127 NPs in water dispersion was obtained. Similarly, TPATCN@F127 NPs in $\mathrm{D}_{2} \mathrm{O}$ could be obtain by replacing DI water with $\mathrm{D}_{2} \mathrm{O}$ during the process.

\section{Nonlinear optical spectra measurement}

A $1300 \mathrm{~nm}$-fs laser (200 fs, $400 \mathrm{kHz}$ ) was focused on a cuvette, which contained TPATCN in $\mathrm{CHCl}_{3}$ solution or TPATCN@F-127 NPs in $\mathrm{D}_{2} \mathrm{O}$ dispersion, via a lens (focal length: $5 \mathrm{~cm}$ ). The laser was generated from an optical parametric amplifier (Orpheus) pumped by a $1040 \mathrm{~nm}$ PHAROS-10W fs laser (10 W), in which the emission wavelength can be tuned in the range of 1040-2600 $\mathrm{nm}$. The nonlinear optical emission was collected from the laser-incident direction by an objective $(20 \times 1.05 \mathrm{NA})$ and recorded with an optical fiber spectrometer (PG 2000, Ideaoptics Instruments) after passing through a short-pass filter.

\section{Measurement of power dependence and three-photon absorption cross section}

The power dependence and three-photon absorption cross section were measured with an upright scanning microscope (BX61 + FV1200, Olympus) equipped with the laser used in the experiment for nonlinear optical spectra measurement.

To measure the excitation power dependence relationship of the fluorescence from TPATCN@F-127 NPs under 1300-nm excitation, a capillary containing TPATCN@F-127 NPs in $\mathrm{D}_{2} \mathrm{O}$ dispersion was imaged via the microscopy system. The excitation wavelength was set at $1300 \mathrm{~nm}$, and the images were captured with various excitation power (the output powers were set as 173 $\mathrm{mW}, 199 \mathrm{~mW}, 222 \mathrm{~mW}, 250 \mathrm{~mW}, 275 \mathrm{~mW}, 301 \mathrm{~mW}, 336 \mathrm{~mW}$ and $363 \mathrm{~mW}$ ). Then, the relationship between fluorescence intensity and excitation power was calculated.

To measure the three-photon absorption cross section, a capillary containing TPATCN in $\mathrm{CHCl}_{3}$ solution was imaged via the microscopic system. The images at $1300-\mathrm{nm}$ excitation and 1550-nm excitation with the same power were captured. The threephoton absorption cross-section of TPATCN was calculated according to the following equation: 45

$$
\frac{\delta_{1}}{\delta_{0}}=\frac{F_{1} \eta_{0} c_{0} n_{0}}{F_{0} \eta_{1} c_{1} n_{1}}
$$

where $\delta$ is the three-photon absorption cross section, $F$ is the three-photon fluorescence intensity, $\eta$ is the $Q Y, c$ is the molar concentration, $\mathrm{n}$ is the refractive index of the solvent, and the subscripts 0 and 1 represent the reference (at $1550 \mathrm{~nm}$ ) and the sample (at $1300 \mathrm{~nm}$ ). Herein, $\eta_{0}=\eta_{1}, c_{0}=c_{1}, n_{0}=n_{1}$. Therefore, the formula could be simplified as follow:

$$
\frac{\delta_{1}}{\delta_{0}}=\frac{F_{1}}{F_{0}}
$$

where $\delta_{0}$ is $5.77 \times 10^{-79} \mathrm{~cm}^{6} \mathrm{~s}^{2}$.

\section{Three-photon fluorescence microscopic system}

For the 3PM of the mouse brain, we used a home-built laser scanning microscope and a $1300 \mathrm{~nm}$ fs laser (maximal output power $400 \mathrm{~mW}, 400 \mathrm{kHz}, 35 \mathrm{fs}$ ) from a noncollinear optical parametric amplifier (Spectra Physics) pumped by a regenerative amplifier (Spirit-16, Spectra Physics). We used a home-built dispersion compensation unit to maintain short pulse durations (50 $\mathrm{fs}$ ) at the sample plane. The maximum laser power after the objective was measured to be $35 \mathrm{~mW}$. Detection included proper filters (FF01593/LP-30-D \& F01-940/SP-30-D, Semrock) and PMT (H107740, Hamamatsu). Data acquisition was based on Scanlmage. ${ }^{46}$

\section{Animals}

All animal experiments were approved by EMBL's Institutional Animal Care and Use Committee. 8-week old female C57 mice were used for in vivo 3PM imaging of brain vasculature.

\section{In vivo three-photon microscopy of cortical vasculature of mice}

To obtain optical access to the brain, a cranial window was implanted as previously described. ${ }^{47}$ During surgery animals were anesthetized with $5 \%$ isoflurane vapor mixed with $\mathrm{O}_{2}$ for induction and maintained at $1-1.5 \%$. For cranial window preparations, a $7 \mathrm{~mm}$ diameter craniotomy was made over the motor cortex, centered at $0.6 \mathrm{~mm}$ posterior and $0.7 \mathrm{~mm}$ lateral to the Bregma point, and a 7-mm coverslip ( $170 \mu \mathrm{m}$ thick) was placed on top of the brain. 
Before imaging experiments, The TPATCN@F-127 NPs in PBS $(200 \mu \mathrm{L}, 1 \mathrm{mg} / \mathrm{mL})$ were injected into the circulatory system of the mice through the tail vein. Then, the mice were fixed and put under the microscope.

The image stack was acquired with a depth interval of $5 \mu \mathrm{m}$, and pixel dwell time was $10 \mu \mathrm{s}, 5$ frames were averaged. The exciation power was exponentially increased with depth and the maximum average power under the objective was $35 \mathrm{~mW}$.

During imaging experiments, mice were anesthetized with isoflurane ( $2 \%$ in oxygen, Harvard Apparatus) and positioned onto a small animal physiological monitoring System (ST2 75-1500, Harvard Apparatus), which allows to maintain animal body temperature at $37.5^{\circ}$ C. During experiments eyes were covered with eye ointment.

\section{Data quantification}

All imaging data were analyzed with Image J software that was developed by National Institutes of Health (Bethesda, Maryland). 3D image reconstruction was performed in Imaris (Bitplane).

\section{Acknowledgement}

This work was supported by National Natural Science Foundation of China (NSFC) (Grant Nos. 61735016, 82001877 and 61975172); China Postdoctoral Science Foundation funded project (Nos. BX20190131, 2019M662633); Zhejiang Provincial Natural Science Foundation of China (LR17F050001); Funding for Postdoctoral Innovation Research Post in Hubei Province. Fundamental Research Funds for the Central Universities (2020-KYY-511108-0007). Work at the EMBL was supported by the European Commission (EU grant FET-PROACTIVE, No. 951991 'Brainiaqs'). The authors also thank Dr. Senthilkumar Deivasigamani for the surgeries and Prof. Cornelius Gross for support.

\section{Conflicts of interest}

There are no conflicts to declare.

\section{References}

1. N. Ji, J. Freeman and S. L. Smith, Technologies for imaging neural activity in large volumes, Nat Neurosci, 2016, 19, 1154-1164.

2. G. S. Hong, A. L. Antaris and H. J. Dai, Near-infrared fluorophores for biomedical imaging, Nat Biomed Eng, $2017,1$.

3. C. W. T. Leung, Y. N. Hong, S. J. Chen, E. G. Zhao, J. W. Y. Lam and B. Z. Tang, A Photostable AIE Luminogen for Specific Mitochondrial Imaging and Tracking, Journal Of the American Chemical Society, 2013, 135, 62-65.

4. G. Silasi, D. Xiao, M. P. Vanni, A. C. Chen and T. H. Murphy, Intact skull chronic windows for mesoscopic wide-field imaging in awake mice, J Neurosci Methods, 2016, 267, 141-149.

5. J. L. Fan, J. A. Rivera, W. Sun, J. Peterson, H. Haeberle, S. Rubin and N. Ji, High-speed volumetric two-photon fluorescence imaging of neurovascular dynamics, Nat Commun, 2020, 11, 6020.

6. K. Kisler, D. Lazic, M. D. Sweeney, S. Plunkett, M. El Khatib, S. A. Vinogradov, D. A. Boas, S. Sakadzic and B. V. Zlokovic, In vivo imaging and analysis of cerebrovascular hemodynamic responses and tissue oxygenation in the mouse brain, Nat Protoc, 2018, 13, 1377-1402.

7. W. Heeman, W. Steenbergen, G. M. van Dam and E. C. Boerma, Clinical applications of laser speckle contrast imaging: a review, $J$ Biomed Opt, 2019, 24, 080901.

8. W. Feng, S. Liu, C. Zhang, Q. Xia, T. Yu and Z. Dan, Comparison of cerebral and cutaneous microvascular dysfunction with the development of type 1 diabetes Theranostics, 2019, 9, 5854-5868.

9. J. L. Geng, C. C. Goh, W. Qin, R. R. Liu, N. Tomczak, L. G. Ng, B. Z. Tangde and B. Liu, Silica shelled and block copolymer encapsulated red-emissive AIE nanoparticles with 50\% quantum yield for two-photon excited vascular imaging, Chemical communications, 2015, 51, 13416-13419.

10. R. D. Dorand, D. S. Barkauskas, T. A. Evans, A. Petrosiute and A. Y. Huang, Comparison of intravital thinned skull and cranial window approaches to study CNS immunobiology in the mouse cortex, Intravital, 2014, 3, e21978.

11. T. Jiang, D. Y. Li, Y. D. Hang, Y. T. Gao, H. Q. Zhang, X. Y. Zhao, X. Li, B. Li, J. Qian and J. L. Hua, Tetraphenylethene end-capped diketopyrrolopyrrole fluorogens with AIE and large two-photon absorption cross-sections features and application in bioimaging, Dyes Pigments, 2016, 133, 201-213.

12. D. Y. Li, L. Xue, Z. F. Zhu, X. Y. Zhao and J. Qian, Graphene oxide nanoparticles for two-photon fluorescence imaging of zebrafish, Opt Quant Electron, 2016, 48, 519.

13. N. Alifu, Z. Z. Sun, A. Zebibula, Z. G. Zhu, X. Y. Zhao, C. F. Wu, Y. L. Wang and J. Qian, Deep-red polymer dots with bright two-photon fluorescence and high biocompatibility for in vivo mouse brain imaging, Opt Commun, 2017, 399, 120-126.

14. H. J. Liu, X. Q. Deng, S. Tong, C. He, H. Cheng, Z. W. Zhuang, M. Y. Gan, J. Li, W. X. Xie, P. Qiu and K. Wang, In Vivo Deep-Brain Structural and Hemodynamic Multiphoton Microscopy Enabled by Quantum Dots, Nano Lett, 2019, 19, 5260-5265.

15. N. G. Horton, K. Wang, D. Kobat, C. G. Clark, F. W. Wise, C. B. Schaffer and C. Xu, In vivo three-photon microscopy of subcortical structures within an intact mouse brain, Nat Photonics, 2013, 7, 205-209.

16. T. Y. Wang and C. Xu, Three-photon neuronal imaging in deep mouse brain, Optica, 2020, 7, 947-960. 
17. H. Y. Tian, D. Y. Li, X. Tang, Y. B. Zhang, Z. Y. Yang, J. Qian, Y. Q. Dong and M. Han, Efficient red luminogen with aggregation-induced emission for in vivo three-photon brain vascular imaging, Mater Chem Front, 2020, 4, 1634-1642.

18. H. W. Ni, Z. C. Xu, D. Y. Li, M. Chen, B. Z. Tang and J. Qian, Aggregation-induced emission luminogen for in vivo three-photon fluorescence lifetime microscopic imaging, J Innov Opt Heal Sci, 2019, 12, 1940005.

19. T. Y. Wang, D. G. Ouzounov, C. Y. Wu, N. G. Horton, B. Zhang, C. H. Wu, Y. P. Zhang, M. J. Schnitzer and C. Xu, Three-photon imaging of mouse brain structure and function through the intact skull, Nat Methods, 2018, 15, 789-792.

20. K. D. Wegner and N. Hildebrandt, Quantum dots: bright and versatile in vitro and in vivo fluorescence imaging biosensors, Chem Soc Rev, 2015, 44, 4792-4834.

21. T. W. Chen, T. J. Wardill, Y. Sun, S. R. Pulver, S. L. Renninger, A. Baohan, E. R. Schreiter, R. A. Kerr, M. B. Orger, V. Jayaraman, L. L. Looger, K. Svoboda and D. S. Kim, Ultrasensitive fluorescent proteins for imaging neuronal activity, Nature, 2013, 499, $295-300$.

22. S. Q. He, J. Song, J. L. Qu and Z. Cheng, Crucial breakthrough of second near-infrared biological window fluorophores: design and synthesis toward multimodal imaging and theranostics, Chem Soc Rev, 2018, 47, 4258-4278.

23. A. C. Grimsdale, K. L. Chan, R. E. Martin, P. G. Jokisz and A. B. Holmes, Synthesis of Light-Emitting Conjugated Polymers for Applications in Electroluminescent Devices, Chem Rev, 2009, 109, 897-1091.

24. M. Shimizu and T. Hiyama, Organic fluorophores exhibiting highly efficient photoluminescence in the solid state, Chem Asian J, 2010, 5, 1516-1531.

25. Z. T. Deng, O. Schulz, S. Lin, B. Q. Ding, X. W. Liu, X. X. Wei, R. Ros, H. Yan and Y. Liu, Aqueous Synthesis of Zinc Blende CdTe/CdS Magic-Core/Thick-Shell Tetrahedral-Shaped Nanocrystals with Emission Tunable to Near-Infrared, J Am Chem Soc, 2010, 132, 55925593.

26. S. Kim, Y. T. Lim, E. G. Soltesz, A. M. De Grand, J. Lee, A. Nakayama, J. A. Parker, T. Mihaljevic, R. G. Laurence, D. M. Dor, L. H. Cohn, M. G. Bawendi and J. V. Frangioni, Near-infrared fluorescent type II quantum dots for sentinel lymph node mapping, Nat Biotechnol, 2004, 22, 93-97.

27. J. D. Luo, Z. L. Xie, J. W. Y. Lam, L. Cheng, H. Y. Chen, C. F. Qiu, H. S. Kwok, X. W. Zhan, Y. Q. Liu, D. B. Zhu and B. Z. Tang, Aggregationinduced emission of 1-methyl-1,2,3,4,5-pentaphenylsilole, Chem Commun, 2001, 1740-1741.

28. B. Z. Tang, X. W. Zhan, G. Yu, P. P. S. Lee, Y. Q. Liu and D. B. Zhu, Efficient blue emission from siloles, J Mater Chem, 2001, 11, 29742978.

29. R. Hu, N. L. C. Leung and B. Z. Tang, AIE macromolecules: syntheses, structures and functionalities, Chem Soc Rev, 2014, 43, 44944562.

30. D. Y. Li, W. Qin, B. Xu, J. Qian and B. Z. Tang, AIE Nanoparticles with High Stimulated Emission Depletion Efficiency and Photobleaching Resistance for Long-Term Super-Resolution Bioimaging, Adv Mater, 2017, 29, 1703643.

31. M. X. Liu, B. B. Gu, W. B. Wu, Y. K. Duan, H. J. Liu, X. Q. Deng, M. Z. Fan, X. M. Wang, X. B. Wei, K. T. Yong, K. Wang, G. X. Xu and B. Liu, Binary Organic Nanoparticles with Bright Aggregation-Induced Emission for Three-Photon Brain Vascular Imaging, Chem Mater, 2020, 32, 6437-6443.

32. Y. L. Wang, M. Chen, N. Alifu, S. W. Li, W. Qin, A. J. Qin, B. Z. Tang and J. Qian, Aggregation-Induced Emission Luminogen with DeepRed Emission for Through-Skull Three-Photon Fluorescence Imaging of Mouse, Acs Nano, 2017, 11, 10452-10461.

33. Y. L. Wang, X. Han, W. Xi, J. Y. Li, A. W. Roe, P. Lu and J. Qian, Bright AIE Nanoparticles with F127 Encapsulation for Deep-Tissue ThreePhoton Intravital Brain Angiography, Adv Healthc Mater, 2017, 6, 1700685.

34. Y. Wang, V. D. Ta, Y. Gao, T. C. He, R. Chen, E. Mutlugun, H. V. Demir and H. D. Sun, Stimulated Emission and Lasing from CdSe/CdS/ZnS Core-Multi-Shell Quantum Dots by Simultaneous Three-Photon Absorption, Adv Mater, 2014, 26, $2954-2961$.

35. L. C. Cheng, N. G. Horton, K. Wang, S. J. Chen and C. Xu, Measurements of multiphoton action cross sections for multiphoton microscopy, Biomedical Optics Express, 2014, 5, 3427-3433.

36. Z. R. Ma, M. X. Zhang, J. Y. Yue, C. Alcazar, Y. T. Zhong, T. C. Doyle, H. J. Dai and N. F. Huang, Near-Infrared IIb Fluorescence Imaging of Vascular Regeneration with Dynamic Tissue Perfusion Measurement and High Spatial Resolution, Adv Funct Mater, $2018,28,1803417$.

37. Y. T. Zhong, Z. R. Ma, F. F. Wang, X. Wang, Y. J. Yang, Y. L. Liu, X. Zhao, J. C. Li, H. T. Du, M. X. Zhang, Q. H. Cui, S. J. Zhu, Q. C. Sun, H. Wan, Y. Tian, Q. Liu, W. Z. Wang, K. C. Garcia and H. J. Dai, In vivo molecular imaging for immunotherapy using ultra-bright nearinfrared-IIb rare-earth nanoparticles, Nat Biotechnol, 2019, 37, 1322-1331.

38. M. X. Zhang, J. Y. Yue, R. Cui, Z. R. Ma, H. Wan, F. F. Wang, S. J. Zhu, Y. Zhou, Y. Kuang, Y. T. Zhong, D. W. Pang and H. J. Dai, Bright quantum dots emitting at similar to $1,600 \mathrm{~nm}$ in the NIR-IIb window for deep tissue fluorescence imaging, $P$ Natl Acad Sci USA, 2018, 115, 6590-6595.

39. D. Y. Li, Z. Zheng, T. T. Yu, B. Z. Tang, P. Fei, J. Qian and D. Zhu, Visible-near infrared-Il skull optical clearing window for in vivo cortical vasculature imaging and targeted manipulation, J Biophotonics, 2020, 133, e202000142.

40. A. L. Antaris, H. Chen, K. Cheng, Y. Sun, G. Hong, C. Qu, S. Diao, Z. Deng, X. Hu, B. Zhang, X. Zhang, O. K. Yaghi, Z. R. Alamparambil, X. Hong, Z. Cheng and H. Dai, A small-molecule dye for NIR-II imaging, Nat Mater, 2016, 15, 235-242.

41. J. Qi, C. W. Sun, D. Y. Li, H. Q. Zhang, W. B. Yu, A. Zebibula, J. W. Y. Lam, W. Xi, L. Zhu, F. H. Cai, P. F. Wei, C. L. Zhu, R. T. K. Kwok, L. L. Streich, R. Prevedel, J. Qian and B. Z. Tang, Aggregation-Induced Emission Luminogen with Near-Infrared-II Excitation and NearInfrared-I Emission for Ultradeep Intravital Two-Photon Microscopy, Acs Nano, 2018, 12, 7936-7945.

42. D. Kobat, N. G. Horton and C. Xu, In vivo two-photon microscopy to 1.6-mm depth in mouse cortex, J Biomed Opt, $2011,16,106014$.

43. K. Lingjie; and C. M., A high throughput (>90\%), large compensation range, single-prism femtosecond pulse compressor, arXiv, 2013, 1306.5011.

44. D. Wang, J. Qian, S. L. He, J. S. Park, K. S. Lee, S. H. Han and Y. Mu, Aggregation-enhanced fluorescence in PEGylated phospholipid nanomicelles for in vivo imaging, Biomaterials, 2011, 32, 5880-5888. 
bioRxiv preprint doi: https://doi.org/10.1101/2020.12.25.424420; this version posted December $26,2020$. The copyright holder for this

preprint (which was not certified by peer review) is the author/funder. All rights reserved. No reuse allowed without permission.

45. D. A. Oulianov, I. V. Tomov, A. S. Dvornikov and P. M. Rentzepis, Observations on the measurement of two-photon absorption crosssection, Opt Commun, 2001, 191, 235-243.

46. T. A. Pologruto, B. L. Sabatini and K. Svoboda, Scanlmage: Flexible software for operating laser scanning microscopes, Biomed Eng Online, 2003, 2.

47. A. Holtmaat, T. Bonhoeffer, D. K. Chow, J. Chuckowree, V. De Paola, S. B. Hofer, M. Hubener, T. Keck, G. Knott, W. C. A. Lee, R. Mostany, T. D. Mrsic-Flogel, E. Nedivi, C. Portera-Cailliau, K. Svoboda, J. T. Trachtenberg and L. Wilbrecht, Long-term, high-resolution imaging in the mouse neocortex through a chronic cranial window, Nat Protoc, 2009, 4, 1128-1144. 